\title{
TWO-STEP RUNGE-KUTTA METHODS AND HYPERBOLIC PARTIAL DIFFERENTIAL EQUATIONS
}

\author{
R. A. RENAUT
}

\begin{abstract}
The purpose of this study is the design of efficient methods for the solution of an ordinary differential system of equations arising from the semidiscretization of a hyperbolic partial differential equation. Jameson recently introduced the use of one-step Runge-Kutta methods for the numerical solution of the Euler equations. Improvements in efficiency up to $80 \%$ may be achieved by using two-step Runge-Kutta methods instead of the classical onestep methods. These two-step Runge-Kutta methods were first introduced by Byrne and Lambert in 1966. They are designed to have the same number of function evaluations as the equivalent one-step schemes, and thus they are potentially more efficient. By solving a nonlinear programming problem, which is specified by stability requirements, optimal two-step schemes are designed. The optimization technique is applicable for stability regions of any shape.
\end{abstract}

\section{INTRODUCTION}

In this paper we consider a class of pseudo-Runge-Kutta methods for the solution of an ordinary differential system of equations

$$
y^{\prime}=f(y)
$$

which arises from the semidiscretization of a hyperbolic partial differential equation. In 1982, Jameson [7] initiated interest in the use of one-step Runge-Kutta methods for the numerical solution of the Euler equations. He applied the van der Houwen [4] optimal schemes in codes for the solution of the Euler equations by central differences. These schemes are optimal because they have regions of stability enclosing a maximal interval on the imaginary axis, as is required when central differences are used for the semidiscretization. Here we demonstrate that greater efficiency is achieved by using two- rather than onestep Runge-Kutta formulae. These Runge-Kutta methods were first considered by Byrne and Lambert [1] in 1966.

We define an explicit two-step $m$-stage Runge-Kutta method as

$$
y_{n+1}=(1-\beta) y_{n}+\beta y_{n-1}+h \sum_{i=1}^{m}\left(v_{i} f\left(y_{n-1}^{i}\right)+w_{i} f\left(y_{n}^{i}\right)\right) \text {, }
$$

Received January 26, 1989; revised September 21, 1989.

1980 Mathematics Subject Classification (1985 Revision). Primary 65M20, 65M10, 65M05.

Key words and phrases. Pseudo-Runge-Kutta methods, stability, hyperbolic partial differential equations, method of lines. 
where

$$
y_{m}^{i}=\left\{\begin{array}{l}
y_{m}, \quad i=1, \\
y_{m}+h \sum_{j=1}^{i-1} a_{i j} f\left(y_{m}^{j}\right), \quad i>1
\end{array}\right.
$$

The vector $y_{n}$ represents a numerical approximation to the analytical solution $y(t)$ at $t=t_{n}$, and $h$ is the step length, $t_{n+1}=t_{n}+h$. Observe that the schemes considered by Byrne and Lambert [1] have $\beta=0$. It is clear that the form of the $y_{n}^{i}$ and $y_{n-1}^{i}$ means that no more function evaluations are required for two steps than for the equivalent one-step formula. This is because the function evaluations at the time $t_{n-1}$ are the same as those taken at time $t_{n}$ in the previous step. Therefore, provided that information is stored from step to step, the two-step schemes are potentially as efficient as the one-step methods when working with a constant stepsize. Furthermore, the number of degrees of freedom of the two-step method is $\frac{1}{2} m(m+3)+1$ as compared to $\frac{1}{2} m(m+1)$ for the one-step method. Thus an $m$-stage two-step scheme has the same flexibility as an $(m+1)$-stage one-step scheme. For a large number of stages, the difference is negligible but typically one only uses a few stages, and then the extra flexibility is useful. Here we take advantage of this flexibility to design schemes with optimal stability regions with respect to given domains in the complex plane. For our analysis we will consider the scalar problem $y^{\prime}=f(y)$, where $y$ is scalar. The results immediately extend to the case of systems.

In the next section we develop the order conditions for the two-step formulae using Butcher series. For a given number of stages, higher order is attainable than with one-step schemes. Byrne and Lambert [1] presented a two-stage scheme of order three and a three-stage scheme of order four. With four stages no improvement is possible and order four is attained.

In order to design efficient schemes for hyperbolic problems, the stability properties of these schemes must be studied. This is done in $\S 2$, where a review of results about maximal stability intervals for Runge-Kutta methods is also presented. We develop criteria for the stability of the two-step schemes and use these to prove that the maximal interval of stability on the imaginary axis for a two-step two-stage order-three scheme is 1.0. In other cases, optimal schemes are found numerically. The strategy to determine optimal schemes is described in the last section. Further explanation is given by Renaut [14].

The results show that generally two-step schemes are more efficient than their one-step counterparts. To enable reasonable comparison, we present scaled results that take into account the number of function evaluations being performed. With three stages and order three, an improvement of $64 \%$ is predicted using two-step schemes for a centrally differenced hyperbolic problem. The optimization procedure is applied not only for intervals of stability on the imaginary axis but also for stability within regions in the complex left-half plane. This is of interest when the hyperbolic equation is discretized with forward or backward 
differences instead of central differences. Improvement in efficiency up to $80 \%$ is predicted for the cases considered here.

Observe that this optimization technique may be applied for any regions in the complex plane and can therefore be used to determine optimal schemes for any semidiscretizations.

\section{ORDER CONDITIONS AND BUTCHER SERIES}

First we review the conditions for the convergence of numerical solutions of (0.2). As in Henrici [3], the method (0.2) is said to be convergent only if, for all Lipschitz functions $f$, the solution $y(t)$ of the initial value problem $y^{\prime}=f(y)$, $y(0)=y_{0}$, defined on the interval $t \in[0, \tau)$ satisfies

$$
\lim _{\substack{n \rightarrow \infty \\ n h=t}} y_{n}=y(t)
$$

Now the multistage method $(0.2)$ is associated with a nonlinear difference operator $y_{n+1}-Z\left(y_{n}, y_{n-1}\right)$, where $Z$ denotes the operators on the right-hand side of equation (0.2). The method is said to be accurate of order $p$, at $t=t_{n}$, if $p$ is the largest integer such that

$$
y\left(t_{n+1}\right)-Z\left(y\left(t_{n}\right), y\left(t_{n-1}\right)\right)=O\left(h^{p+1}\right), \quad|h| \ll 1 .
$$

If $p \geq 1$, the method is consistent. Further, the method is zero-stable if no root of the polynomial $\rho(\alpha)$, defined by

$$
\rho(\alpha)=\alpha^{2}-(1-\beta) \alpha-\beta,
$$

has modulus greater than one, and if a root has modulus one it must be simple. Then the method is convergent if and only if it is zero-stable and consistent [3]. Therefore, it is convergent only if $-1<\beta \leq 1$ since $\rho(\alpha)$ has roots $\alpha_{1}=1$ and $\alpha_{2}=-\beta$. Furthermore, if we expand $y\left(t_{n+1}\right)$ and $y\left(t_{n-1}\right)$ about $y\left(t_{n}\right)$, using Taylor's theorem, we see that

$$
\begin{aligned}
\lim _{h \rightarrow 0}[ & \left.y\left(t_{n+1}\right)-Z\left(y\left(t_{n}\right), y\left(t_{n-1}\right)\right)\right] \\
= & h\left[(1+\beta) y^{\prime}\left(t_{n}\right)-\sum_{i=1}^{m}\left(v_{i}+w_{i}\right) f\left(y_{n}\right)\right]+O\left(h^{2}\right) .
\end{aligned}
$$

Thus consistency requires

$$
1+\beta=\sum_{i=1}^{m}\left(v_{i}+w_{i}\right)
$$

and convergence

$$
\sum_{i=1}^{m}\left(v_{i}+w_{i}\right) \neq 0 .
$$

In general, order of accuracy greater than one is desired. Order conditions can be derived in a variety of ways. The tensor notation used by Henrici [3] was applied by Renaut [14] to derive order conditions up to order four. Here 
we use Butcher series and follow the approach of Hairer and Wanner [2] to get a formula from which order conditions up to any order of accuracy can be derived. Jackiewicz, Renaut, and Feldstein [6] also used Butcher series to get the order conditions for implicit two-step methods but without the use of the composition theorem of Hairer and Wanner [2]. To apply the theory of Hairer and Wanner [2], we need to write the method $(0.2)$ in the matrix-vector form

$$
y=A^{(0)} y_{0}+h A^{(1)} f(y) \text {. }
$$

Here $A^{(0)}$ and $A^{(1)}$ are fixed $(2 m+2) \times(2 m+2)$ matrices, and $y, y_{0}$, and $f(y)$ are $(2 m+2)$-dimensional vectors, where $f$ acts on the vector $y$ componentwise. In this case,

and

$$
A^{(0)}=m\left\{\left(\begin{array}{ccccccccc}
0 & \ldots & 0 & 1 & 0 & \ldots & 0 & 0 & 0 \\
\vdots & & \vdots & \vdots & \vdots & & \vdots & \vdots & \vdots \\
0 & \ldots & 0 & 1 & 0 & \ldots & 0 & 0 & 0 \\
0 & \ldots & 0 & 1 & 0 & \ldots & 0 & 0 & 0 \\
0 & \ldots & 0 & 0 & 0 & \ldots & 0 & 1 & 0 \\
\vdots & & \vdots & \vdots & & & \vdots & \vdots & \vdots \\
0 & \ldots & 0 & 0 & 0 & \ldots & 0 & 1 & 0 \\
0 & \ldots & 0 & \beta & 0 & \ldots & 0 & 1-\beta & 0
\end{array}\right)\right.
$$

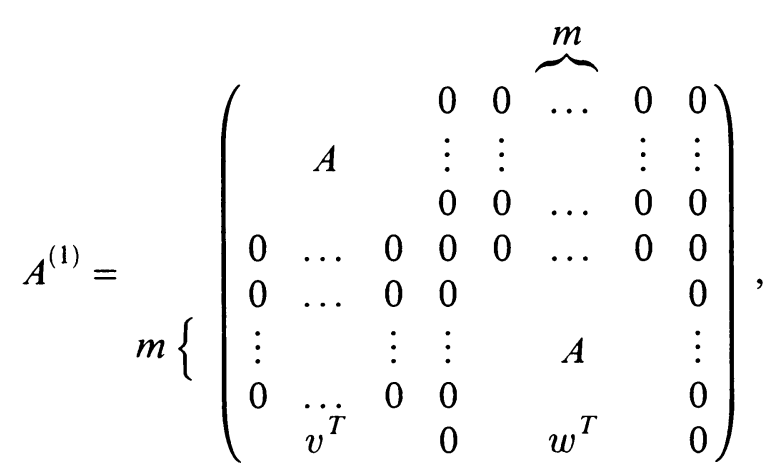

where

$$
A=\left(\begin{array}{cccc}
0 & \ldots & \ldots & 0 \\
a_{21} & 0 & \ldots & 0 \\
\vdots & \ddots & \ddots & \vdots \\
a_{m 1} & \ldots & a_{m, m-1} & 0
\end{array}\right)
$$

$v^{T}=\left(v_{1}, v_{2}, \ldots, v_{m}\right)^{T}$, and $w^{T}=\left(w_{1}, w_{2}, \ldots, w_{m}\right)^{T}$. Also, $y_{0}$ is defined by

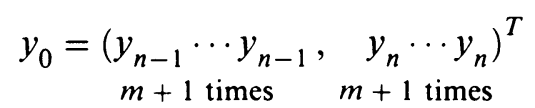

and $y=\left(y_{n-1}^{1}, y_{n-1}^{2}, \ldots, y_{n-1}^{m}, y_{n}, y_{n}^{1}, \ldots, y_{n}^{m}, y_{n+1}\right)^{T}$. These definitions of $A^{(0)}, A^{(1)}, y_{0}$, and $y$ are not unique; see [6] for a different definition. 
We assume for $y$ a Butcher series

$$
y=\sum_{t \in \bar{T}} \frac{h^{r}}{r !} \Phi(t) F(t)\left(y_{0}\right)=B\left(\Phi, y_{0}\right),
$$

where $\bar{T}$ is the set of all monotonically labelled trees, $r$ is the order of the tree, and

$$
\Phi(t)=\left(\Phi_{1}(t), \ldots, \Phi_{2 m+2}(t)\right)^{T}
$$

is unknown. $F(t)$ is the differential associated with tree $t$ such that

$$
F(\tau)=f, \quad \tau=\bullet,
$$

and

$$
F(t)=f^{m} \bullet\left(F\left(t_{1}\right), F\left(t_{2}\right), \ldots, F\left(t_{m}\right)\right)
$$

if $t=\left[t_{1}, t_{2}, \ldots, t_{m}\right]$ is the rooted tree such that if its root is removed, the remaining subtrees are just $t_{1}, t_{2}, \ldots, t_{m}$. Here, $\tau=\bullet$ is the tree of order 1 , and the composition $F(t)=f^{m} \bullet\left(F\left(t_{1}\right), F\left(t_{2}\right), \ldots, F\left(t_{m}\right)\right)$ means that the differential $f^{m}$ acts on each of the differentials $F\left(t_{1}\right), F\left(t_{2}\right), \ldots, F\left(t_{m}\right)$. The Butcher series for $y_{0}$ is given by $B\left(p, y_{0}\right)$, where

$$
p_{i}(t)= \begin{cases}(-1)^{r}, & i \leq m, \\ 1, & i \geq m+1, t=\varnothing, \\ 0, & \text { otherwise. }\end{cases}
$$

Forming the Butcher series for the numerical method (0.2) and applying the composition theorem of Hairer and Wanner [2] gives

$$
B\left(\Phi, y_{0}\right)=A^{(0)} B\left(p, y_{0}\right)+A^{(1)} B\left(\Phi^{\prime}, y_{0}\right) \text {. }
$$

Comparing terms gives

$$
\Phi(t)=A^{(0)} p(t)+A^{(1)} \Phi^{\prime}(t) .
$$

For order of accuracy $p$, the last component of this equation must agree with the Butcher series of the Taylor polynomial for $y_{n+1}$ up to terms of order $p$. Therefore,

$$
(\Phi(\bar{t}))_{2 m+2}=1\left\{\begin{array}{l}
\forall \bar{t} \in \bar{T} \\
r \leq p .
\end{array}\right.
$$

Application of the Kastlunger Theorem [2] to $\Phi^{\prime}(t)$ gives

$$
\Phi^{\prime}(t)=r\left(\Phi\left(t_{1}\right) \bullet \Phi\left(t_{2}\right) \bullet \cdots \bullet \Phi\left(t_{m}\right)\right)
$$

and thus the order conditions. These are given in Table 1 for order of accuracy up to $p=4$.

The relationship between the number of degrees of freedom and the order attainable for both one- and two-step Runge-Kutta is summarized in Table 2. The maximal order of the five-stage, two-step scheme has not been calculated but we conjecture that it is five. For order six, 37 conditions must be satisfied by 21 coefficients, and our experience with these schemes suggests that this is not 
TABLE 1

Order conditions for two-step Runge-Kutta

\begin{tabular}{|c|c|c|c|}
\hline $\bar{t}$ & $r$ & Differential & Order Conditions \\
\hline 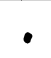 & 1 & $f(t)$ & $1=-\beta+\left(v^{T}+w^{T}\right) \mathbf{1}$ \\
\hline 1 & 2 & $f^{\prime} \bullet f$ & $1=\beta+2\left(v^{T} b+w^{T} c\right)$ \\
\hline V & 3 & $f^{\prime \prime} \bullet(f, f)$ & $1=-\beta+3\left(v^{T} b^{2}+w^{T} c^{2}\right)$ \\
\hline i & 3 & $f^{\prime} \bullet\left(f^{\prime} \bullet f\right)$ & $1=-\beta+3 v^{T} 1$ \\
\hline & & & $+6\left(\left(v^{T}+w^{T}\right) A_{1} c-v^{T} A_{1} \mathbf{1}\right)$ \\
\hline$\downarrow$ & 4 & $f^{\prime \prime \prime} \bullet(f, f, f)$ & $1=\beta+4\left(v^{T} b^{3}+w^{T} c^{3}\right)$ \\
\hline$V$ & 4 & $f^{\prime \prime} \bullet\left(f,\left(f^{\prime} \bullet f\right)\right)$ & $1=\beta+4 v^{T}\left(b+2\left(b \bullet A_{1} b\right)\right)$ \\
\hline & & & $+4 w^{T}\left(2 c \bullet A_{1} c\right)$ \\
\hline$Y$ & 4 & $f^{\prime} \bullet\left(f^{\prime \prime} \bullet(f, f)\right)$ & $1=\beta+4\left(-v^{T} 1+3 v^{T} A_{1} b^{2}+3 w^{T} A_{1} c^{2}\right)$ \\
\hline & & & $1=\beta-4 v^{T} \mathbf{1}+12 v^{T} c$ \\
\hline & 4 & $f^{\prime} \bullet\left(f^{\prime} \bullet\left(f^{\prime} \bullet f\right)\right)$ & $+24\left(v^{T}+w^{T}\right) A_{1}^{2} c-24 v^{T} A_{1} c$ \\
\hline
\end{tabular}

$$
\begin{gathered}
\mathbf{1}=(1,1, \ldots, 1)^{T} \in \mathbf{R}^{m}, b=\left(b_{1}, b_{2}, \ldots, b_{m}\right)^{T}, b_{i}=c_{i}-1, c=\left(c_{1}, \ldots, c_{m}\right)^{T}, \\
c_{i}=\sum_{i=1}^{i-1} a_{i j}, \quad\left(b^{k}\right)_{i}=b_{i}^{k}, \text { and } b \bullet c \text { is componentwise multiplication. }
\end{gathered}
$$

possible. Except with four stages, one order of accuracy higher, using two steps rather than one, is obtained. In this case there are more remaining degrees of freedom that can be used in a variety of ways. Renaut [14] exploited the degrees of freedom available to design error control schemes. Byrne and Lambert [1] minimized the truncation error.

TABLE 2

Comparison of order for one-and two-step schemes

\begin{tabular}{|c|c|c|c|}
\hline $\begin{array}{c}\text { Number of } \\
\text { stages }\end{array}$ & $\begin{array}{c}\text { Number of } \\
\text { steps }\end{array}$ & $\begin{array}{c}\text { Maximal } \\
\text { Order }\end{array}$ & $\begin{array}{c}\text { Degrees of } \\
\text { Freedom }\end{array}$ \\
\hline 1 & 1 & 1 & 1 \\
1 & 2 & 2 & 3 \\
2 & 1 & 2 & 3 \\
2 & 2 & 3 & 6 \\
3 & 1 & 3 & 6 \\
3 & 2 & 4 & 10 \\
4 & 1 & 4 & 10 \\
4 & 2 & 4 & 15 \\
5 & 1 & 4 & 15 \\
5 & 2 & - & 21 \\
\hline
\end{tabular}




\section{Stability AND STABILITY INTERVALS}

The solution of the linear test equation $y^{\prime}=\lambda y, \lambda \in \mathbf{C}$, by (0.2) yields the recurrence relation

$$
y_{n+1}=S(z) y_{n}+P(z) y_{n-1},
$$

where $z=h \lambda$ and $S(z)$ and $P(z)$ are polynomials of degree $m, S(z)=$ $\sum_{i=0}^{m} s_{i} z^{i}, P(z)=\sum_{i=0}^{m} p_{i} z^{i}$. In terms of the variables in (0.2),

$$
\begin{aligned}
& s_{0}=1-\beta, \quad s_{i}=\sum_{i=1}^{m} w_{i}, \\
& s_{i}=\sum_{k_{1}=i}^{m} \sum_{k_{2}=i-1}^{k_{1}-1} \cdots \sum_{k_{i}=1}^{k_{i-1}-1} a_{k_{1} k_{2}} a_{k_{2} k_{3}} \cdots a_{k_{i-1} k_{i}} w_{k_{1}}, \\
& p_{0}=\beta, \quad p_{1}=\sum_{i=1}^{m} v_{i}, \\
& p_{i}=\sum_{k_{1}=i}^{m} \sum_{k_{2}=i-1}^{k_{1}-1} \cdots \sum_{k_{i}=1}^{k_{i-1}-1} a_{k_{1} k_{2}} a_{k_{2} k_{3}} \cdots a_{k_{i-1} k_{i}} v_{k_{1}} .
\end{aligned}
$$

The characteristic equation of $(0.2)$,

$$
\alpha^{2}-S(z) \alpha-P(z)=0, \quad z=h \lambda,
$$

has roots $\alpha_{1}, \alpha_{2}$ which determine the stability of the method. The stability region $S$ is defined by

$$
S=\left\{z \in \mathbf{C}: \begin{array}{l}
\text { roots } \alpha_{1}, \alpha_{2} \text { of }(2.2) \text { satisfy }\left|\alpha_{1}\right|,\left|\alpha_{2}\right| \leq 1 \\
\text { and } \alpha_{1} \neq \alpha_{2} \text { if }\left|\alpha_{1}\right|=\left|\alpha_{2}\right|=1
\end{array}\right\} .
$$

The numerical solution of a partial differential equation by $(0.2)$ is stable provided that all points in the spectrum of the infinite Toeplitz operator of the semidiscretization multiplied by $h$ lie inside $S$. Since the values of the spectrum are proportional to $1 / \Delta x$, where $\Delta x$ is the grid size in the spatial discretization, the numerical solution is stable provided that the spectral curve multiplied by $h / \Delta x$ lies inside $S$. In order to use large time steps, $h$, as large a multiple as possible of the spectral curve must be inside $S$. Thus, because $\mu$ is proportional to $h / \Delta x$, we need to find the largest possible Courant number $\mu$ for which the numerical method is stable.

Suppose the linear hyperbolic test equation

$$
u_{t}=u_{x}, \quad u=u(x, t)
$$

is solved. A three-point central difference approximation is

$$
\frac{d u_{j}}{d t}=\frac{1}{2 \Delta x}\left(u_{j+1}-u_{j-1}\right),
$$




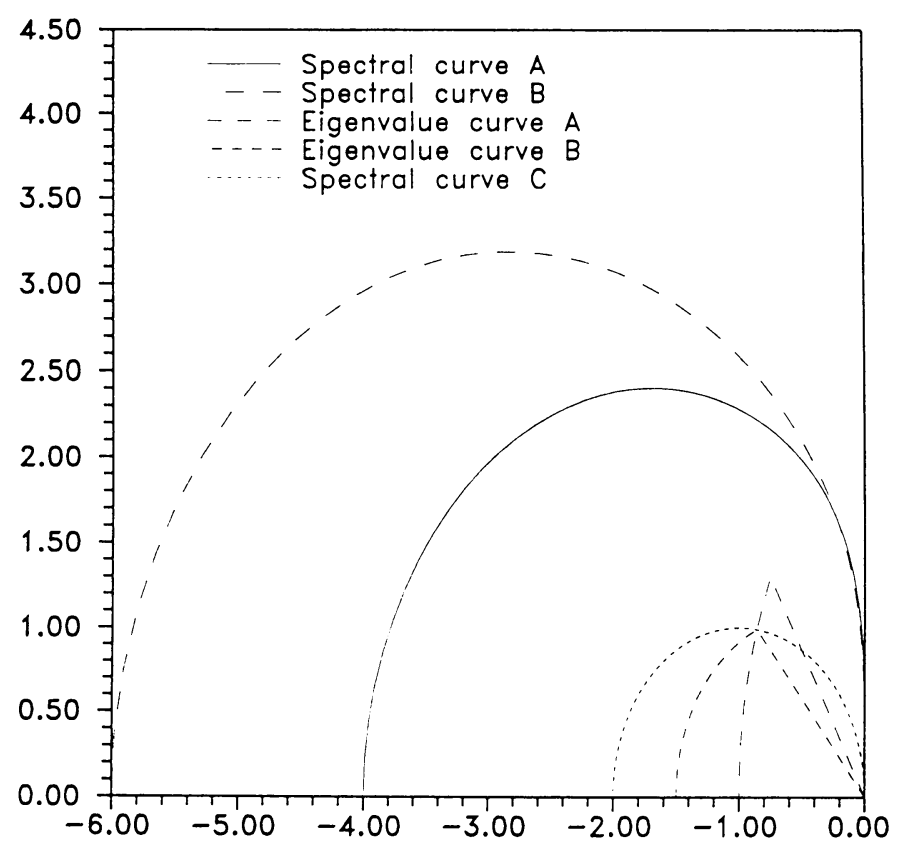

FIGURE 1

Comparison of spectral and eigenvalue curves

where $u_{j}$ approximates $u\left(x_{j}, t\right)$. The spectrum of this approximation is the curve $i \sin \theta, \theta \in[-\pi / 2, \pi / 2]$, which is just the interval [ $-i, i]$ of the imaginary axis. The five-point approximation has spectrum with a larger interval on the imaginary axis. For efficient integration of these methods, methods with stability region enclosing a maximal interval on the imaginary axis are desired. One-step Runge-Kutta methods with this property were first analyzed by van der Houwen [4].

The construction of first-order one-step Runge-Kutta methods which are optimal in the sense of having a stability region enclosing a maximum interval along the imaginary axis can be posed as a minimax problem. The maximum interval of stability along the imaginary axis, $\beta_{\text {imag }}$, is bounded by $2[\mathrm{~m} / 2]$. This bound was refined by Pike and Roe [13] to give $\beta_{\text {imag }} \leq m-1$. Runge-Kutta methods with an odd number of stages, that attain this bound, are also secondorder accurate. Third-order methods satisfy $\beta_{\text {imag }}=\sqrt{(m-1)^{2}+1}$ and are fourth-order if the number of stages is even; see Kinmark and Gray [10]. Such results for two-step schemes are not known. Jeltsch and Nevanlinna [8] proved that $\beta_{\text {imag }}=m \sin (\pi / 2 m)$ for order-one two-step schemes and an odd number of stages. We demonstrate later that a larger value of $\beta_{\text {imag }}$ can be found by relaxing the assumptions of their theorem. 
Large intervals of stability on the imaginary axis are, however, not always desired. If (2.3) is solved using upwinding:

$$
\mathrm{C}: \frac{d u_{j}}{d t}=\frac{u_{j+1}-u_{j}}{\Delta x}
$$

the spectral curve is $\frac{1}{\Delta x}(\cos \theta-1+i \sin \theta)$, which is shown as curve $C$ in Figure 1. This lies in the left-half complex plane and therefore the stability region must enclose as large a multiple as possible of this curve. Spectral curves for the discretizations

$$
\begin{aligned}
& \text { A: } \frac{2 d u_{j}}{d t}+\frac{d u_{j+1}}{d t}=\frac{1}{\Delta x}\left(\frac{-1}{2} u_{j-1}-2 u_{j}+\frac{5}{2} u_{j+1}\right), \\
& \text { B: } \frac{d u_{j-1}}{d t}-\frac{8 d u_{j}}{d t}-\frac{5 d u_{j+1}}{d t}=\frac{12}{\Delta x}\left(u_{j}-u_{j+1}\right)
\end{aligned}
$$

also lie in $\mathbf{C}^{-}$, as shown in Figure 1. The schemes given by $\mathbf{A}$ and $\mathbf{B}$ were chosen as the only two examples of implicit schemes which have order three, are stable, and are based on a Padé approximation. In fact, the results of Iserles and Williamson [5] demonstrate that there are only three possibilities for such maximally accurate schemes of order three. The third scheme is explicit and also gives a curve on $\mathbf{C}^{-}$. Obviously, many other discretizations also give curves in $\mathbf{C}^{-}$, and thus in each case the stability region must be optimized with respect to that curve. There are no solutions of this problem in the literature for either one- or two-step Runge-Kutta methods. Observe that in examples (2.4), (2.5), and (2.6) the spectrum of the infinite Toeplitz operator is not the same as the spectrum of the finite-dimensional operator. The two are compared in Figure 1 for discretizations A and B. The discrepancy occurs because the underlying Toeplitz operator is not normal, and thus the eigenvalues of the Jacobian matrix do not tend to the spectrum of the infinite-dimensional form.

We seek regions in $\mathbf{C}$ in which the roots of the characteristic equation (2.2) have modulus less than one and attain modulus one on its boundary. By the maximum modulus principle we only need to determine the roots of the characteristic equation along the boundary of the region. To decide if a root satisfies stability, the Cohn-Schur criteria may be used. For equation (2.2) they take the form

$$
\begin{aligned}
& \text { (i) }|P(z)| \leq 1, \\
& \text { (ii) }\left.|| P(z)\right|^{2}-1|\leq| \bar{S}(z) P(z)+S(z) \mid \text {. }
\end{aligned}
$$

With strict inequality the roots of the quadratic equation (2.2) lie inside the unit circle [12].

These criteria are expressed in terms of the polynomial coefficients $\left\{s_{i}, p_{i}\right\}$. Therefore, it is convenient to express the order conditions of Table 1 in terms of $\left\{s_{i}, p_{i}\right\}$. Note that the method is of order $p$ only if one root of the quadratic equation (2.2), the principal root, is an approximation of order $p$ to the exponential:

$$
\alpha_{1}(z)-e^{z}=c z^{p+1}+c\left(|z|^{p+2}\right) .
$$


This gives

$$
\begin{aligned}
& p_{0}+s_{0}=1 \text { order } 1, \\
& p_{1}+s_{1}+s_{0}=2 \text { order } 2, \\
& p_{2}+s_{2}+s_{1}+s_{0} / 2=2 \\
& p_{3}+s_{3}+s_{2}+s_{1} / 2+s_{0} / 6=4 / 3 \text { order } 3 .
\end{aligned}
$$

Up to order two, these conditions are sufficient, but for $p=3$ the coefficient of the $f_{y}^{2} f$ differential,

$$
\left(p_{2}+s_{2}\right) \alpha+\frac{p_{3}+s_{3}}{\delta \alpha}\left((\gamma+\delta)^{2}-\alpha(\gamma+3 \delta)\right)=0
$$

is also required. For convenience we have set $\alpha=a_{21}, \gamma=a_{31}$, and $\delta=a_{32}$.

The solution of these equations for the $m=2$ scheme completely defines the coefficients $\left\{s_{2}, p_{2}\right\}$ in terms of $p_{0}$ :

$$
\begin{array}{ll}
s_{0}=1-p_{0}, & s_{1}=\frac{3+p_{0}}{2}, \quad s_{2}=\frac{5-p_{0}}{12}, \\
p_{1}=\frac{p_{0}-1}{2}, & p_{2}=-s_{2} .
\end{array}
$$

The stability region can thus only be optimized with respect to the variable $p_{0}$, which is constrained by zero stability to satisfy $-1<p_{0} \leq 1$.

Theorem. The maximal region of stability attainable on the imaginary axis by a two-step, two-stage, order-three scheme is given by $\beta_{\text {imag }}=1$. The schemes achieving this bound have

$$
P(z)=\frac{1}{5}-\frac{2}{5} z-\frac{2}{5} z^{2} \text { and } S(z)=\frac{4}{5}+\frac{8}{5} z+\frac{2}{5} z^{2} .
$$

Proof. Set $z=i y$ in the Cohn-Schur criteria and substitute the equations (2.10) in (2.7). This yields

$$
\begin{aligned}
& \text { (i) } \frac{1}{144}\left(5-p_{0}\right)^{2} y^{4}+\frac{1}{12}\left(1+p_{0}\right)\left(3+p_{0}\right) y^{2}-\left(1-p_{0}^{2}\right) \leq 0, \\
& \text { (ii) } y^{2} \leq 24 \frac{\left(1-p_{0}^{2}\right)}{\left(5-p_{0}\right)^{2}}
\end{aligned}
$$

Here, condition (ii) implies (i) when $\left|p_{0}\right|<1$. The maximum value of $y$ that satisfies (ii) is $y=1$ and occurs for $p_{0}=\frac{1}{5}$. Therefore, $\beta_{\text {imag }}=1 . P(z)$ and $S(z)$ follow by substitution of $p_{0}=\frac{1}{5}$ in $(2.10)$.

In general, the equations (2.8) determine the number of degrees of freedom available for stability. Equation (2.9) is just an extra condition on the coefficients $\alpha, \gamma$, and $\delta$. Therefore, the determination of maximal Courant numbers is a nonlinear programming problem given by the Cohn-Schur criteria subject to the linear order conditions (2.8) and the zero stability condition $-1<p_{0} \leq 1$. Note also that the truncation error is normalized by the term $\sum_{i=1}^{m}\left(v_{i}+w_{i}\right)$, which tends to zero as $p_{0}$ tends to -1 . Therefore, $p_{0}$ near 
-1 is not preferred. The optimization procedure described next is unlike that adopted by Lawson [11] for finding optimal one-step fifth-order schemes with six stages.

\section{OPTIMAL STABILITY REgIONS}

Here we present a method for determining optimal stability regions for a given number of stages and order of accuracy for one- or two-step Runge-Kutta methods. The same technique could be used for Runge-Kutta methods with more steps. To do this, the Cohn-Schur criteria for higher-order polynomials would lead to more nonlinear constraints.

Our aim is to find coefficients of a method which has a stability region enclosing a given domain $\Omega$ in $\mathbf{C}^{-}$. In the cases considered here, $\Omega$ is either a wedge bounded by the arc of a circle, or a wedge bounded by a spectral curve. The domain $\Omega$ can be uniformly scaled by multiplying each $z \in \mathbf{C}$ by a constant, $\mu$. We wish to maximize $\mu$ so that $\Omega$ still lies in $S$, but only just lies in $S$. The method for which $\mu$ is largest is optimal. Because of the maximum principle, we only need to check stability along the boundary of the domain. Therefore, we discretize the boundary of $\Omega$ and label the points $z_{j}$, $1 \leq j \leq N$. At each point, stability is required, and therefore the Cohn-Schur criteria must be satisfied. Therefore, from equations (2.7) we get two nonlinear constraints at each point, which gives a total of $2 \mathrm{~N}$ nonlinear constraints. Since stability can be determined completely from the coefficients of the characteristic polynomial, we work with the polynomial coefficients rather than the $\left\{\alpha_{i j}, v_{i}, w_{i}\right\}$ of the method. The latter can be calculated from the polynomial coefficients $\left\{p_{i}, s_{i}, 0 \leq i \leq m\right\}$. Depending on the order, the polynomial coefficients are further linearly constrained by equations (2.8). Zero stability also imposes $-1<p_{0} \leq 1$. This leaves $M$ free variables, where $M \leq 2(m+1)$. Then we get an additional variable which is the scaling factor $\mu$. The size of $\Omega$ depends on $\mu$, and thus the $z_{j}$ also depend on $\mu$. In summary, we have the following optimization problem:

$$
\text { maximize } \mu
$$

subject to

$$
\begin{gathered}
-1<p_{0} \leq 1, \\
\left|P\left(z_{j}\right)\right| \leq 1, \quad 1 \leq j \leq N, \\
\left|\bar{S}\left(z_{j}\right) P\left(z_{j}\right)+S\left(z_{j}\right)\right| \leq\left.|1-| P\left(z_{j}\right)\right|^{2} \mid, \quad 1 \leq j \leq N .
\end{gathered}
$$

$P\left(z_{j}\right)$ and $S\left(z_{j}\right)$ are functions of $X=\left(\mu, X_{1} \cdots X_{M}\right)^{T}$ with $\left\{X_{i} \mid 1 \leq i \leq\right.$ $M\} \subset\left\{p_{i}, s_{i} \mid 0 \leq i \leq m\right\}$.

The above problem was solved for the second- and third-order, two- and three-stage, two-step schemes. For comparison, some optimal one-step schemes were also found, but the optimization was much simpler. Details of the solution process are given by Renaut [14]. In many cases it was difficult to get convergence to a solution with a small residual, even using double precision. 
TABLE 3

Runge-Kutta schemes and their degrees of freedom for optimization

\begin{tabular}{|c|c|c|c|}
\hline $\begin{array}{c}\text { Number of } \\
\text { steps }\end{array}$ & $\begin{array}{c}\text { Number of } \\
\text { stages }\end{array}$ & $\begin{array}{c}\text { Order } \\
p \\
\end{array}$ & Free Variables \\
\hline 2 & 2 & 2 & $p_{0} p_{1} p_{2} \quad \mu$ \\
\hline 2 & 2 & 3 & $p_{0} \quad \mu$ \\
\hline 2 & 3 & 2 & $p_{0} p_{1} p_{2} p_{3} s_{3} \quad \mu$ \\
\hline 2 & 3 & 3 & $p_{0} p_{1} p_{2} p_{3} \quad \mu$ \\
\hline 1 & 2 & 2 & - \\
\hline 1 & 3 & 2 & $r_{3} \quad \mu$ \\
\hline 1 & 4 & 2 & $r_{3} r_{4} \quad \mu$ \\
\hline 1 & 3 & 3 & - \\
\hline 1 & 4 & 3 & $r_{4} \quad \mu$ \\
\hline 1 & 5 & 3 & $r_{4} r_{5} \quad \mu$ \\
\hline
\end{tabular}

TABLE 4

Maximal radii of wedges

\begin{tabular}{|c|c|c|c|c|c|c|c|}
\hline Stages & Steps & Order & $49.1^{\circ}$ & $60^{\circ}$ & $45^{\circ}$ & $90^{\circ}$ & $I$ \\
\hline 2 & 1 & 2 & 1.74 & 1.99 & & & 0 \\
3 & 1 & 2 & 3.02 & 2.82 & & 2.00 \\
4 & 1 & 2 & 4.36 & 4.99 & not & $2 \sqrt{2}$ \\
3 & 1 & 3 & 2.15 & 2.52 & calculated & $\sqrt{3}$ \\
4 & 1 & 3 & 3.13 & 3.09 & & & $2 \sqrt{2}$ \\
5 & 1 & 3 & 4.31 & 4.94 & & & $\sqrt{15}$ \\
2 & 2 & 2 & 2.97 & 3.39 & 2.75 & 1.47 & 1.98 \\
3 & 2 & 2 & 4.91 & 5.09 & 5.92 & 2.29 & 2.93 \\
2 & 2 & 3 & 1.85 & 1.99 & 2.06 & 1.00 & 1.00 \\
3 & 2 & 3 & 2.63 & 2.57 & 3.30 & 2.21 & 2.84 \\
\hline
\end{tabular}

TABLE 5

Maximal radii of wedges scaled by number of stages

\begin{tabular}{|c|c|c|c|c|c|c|c|}
\hline Stages & Steps & Order & $49.1^{\circ}$ & $60^{\circ}$ & $45^{\circ}$ & $90^{\circ}$ & $I$ \\
\hline 2 & 1 & 2 & .870 & .995 & \multirow{4}{*}{\multicolumn{2}{|c|}{$\begin{array}{c}\text { not } \\
\text { calculated }\end{array}$}} & 0 \\
\hline 3 & 1 & 2 & 1.007 & .940 & & & .667 \\
\hline 4 & 1 & 2 & 1.090 & 1.248 & & & .707 \\
\hline 3 & 1 & 3 & .717 & 840 & & & .577 \\
\hline 4 & 1 & 3 & .783 & .773 & & & .707 \\
\hline 5 & 1 & 3 & .862 & .988 & & & .775 \\
\hline 2 & 2 & 2 & 1.485 & 1.695 & 1.375 & .735 & .990 \\
\hline 3 & 2 & 2 & 1.637 & 1.697 & 1.973 & .763 & .977 \\
\hline 2 & 2 & 3 & .925 & .995 & 1.030 & .500 & .500 \\
\hline 3 & 2 & 3 & 877 & .857 & $1.1^{\mathrm{n}}$ & .737 & .947 \\
\hline
\end{tabular}


TABLE 6

Coefficients of optimal schemes

\begin{tabular}{|c|c|c|l|l|}
\hline $\begin{array}{c}\text { Region } \\
\Omega\end{array}$ & & $\begin{array}{l}\text { Imaginary } \\
\text { axis }\end{array}$ & $\begin{array}{l}\text { Wedge } \\
\text { angle } 45^{\circ}\end{array}$ & $\begin{array}{l}\text { Wedge } \\
\text { angle } 90^{\circ}\end{array}$ \\
\hline scheme & coefficient & & & \\
\hline$m=2$ & $p_{0}$ & -.950 & -.8 & .178656 \\
$p=2$ & $p_{1}$ & .025 & -.827186 & .321257 \\
& $p_{2}$ & .006 & -.207100 & .005268 \\
\hline$m=2$ & $p_{0}$ & .2 & -.8 & .2 \\
$p=3$ & & & & \\
\hline & $p_{0}$ & .9 & -.949860 & .132890 \\
$m=3$ & $p_{1}$ & -.039 & -.961077 & .186786 \\
$p=2$ & $p_{2}$ & -.009 & -.218305 & .144745 \\
& $p_{3}$ & -.001 & -.018807 & .035683 \\
& $s_{3}$ & .295 & .019814 & .179877 \\
\hline & $p_{0}$ & 1.00 & .73 & .378628 \\
$m=3$ & $p_{1}$ & 0 & .59 & .430854 \\
$p=3$ & $p_{2}$ & 0 & .14 & .246454 \\
& $p_{3}$ & 0 & .018 & .078024 \\
\hline
\end{tabular}

Many of the solutions apparently converged to a $p_{0}$ near to -1 . In these cases we imposed an additional constraint that $p_{0}$ be greater than some number, for example, -.9 . The optimal schemes then converged to this lower bound for $p_{0}$. In each case, a solution was found for a small value of $N, \operatorname{such}$ as $N=5$. We then increased $N$ and found a new solution with the old solution as the starting value. The process was continued until the solutions from successive problems were the same to the accuracy allowable by the subroutine. Usually only a few iterations were needed to get convergence. We used a variety of NAG and Harwell library routines but obtained best results with the NAG routine E04UBF, for which the results are given here. The schemes investigated are summarized in Table 3. Note that the characteristic polynomial of the one-step Runge-Kutta schemes is denoted by $R(z)$. Observe that we have not calculated results for all the one-step schemes, because differences between the one-step and two-step schemes are adequately shown by the other results.

We used five different domains $\Omega$ in the calculations. One of these was the imaginary axis, the others were wedge shaped regions subtending an angle $2 \alpha$ at the origin and bounded by a smooth curve. For wedges with $\alpha=45^{\circ}$ and $\alpha=90^{\circ}$, the wedge was bounded by the arc of a circle. The other two wedges were bounded by the spectral curves of the Jacobians of the semidiscretizations $A$ and B, equations (2.5) and (2.6). These subtend angles $60^{\circ}$ and $49.1^{\circ}$ at the origin, respectively. In Table 4 we give the maximum radius of the wedge obtained for each scheme. These values, however, do not allow fair comparison, as there is more work in a three-stage scheme than in a two-stage scheme. Thus, Table 5 is the same as Table 4 with the radii scaled by the number of stages.

Observe that the wedge with angle $60^{\circ}$ apparently allows larger Courant numbers than the wedge with angle $49.1^{\circ}$. This is not a problem, as the bounding 


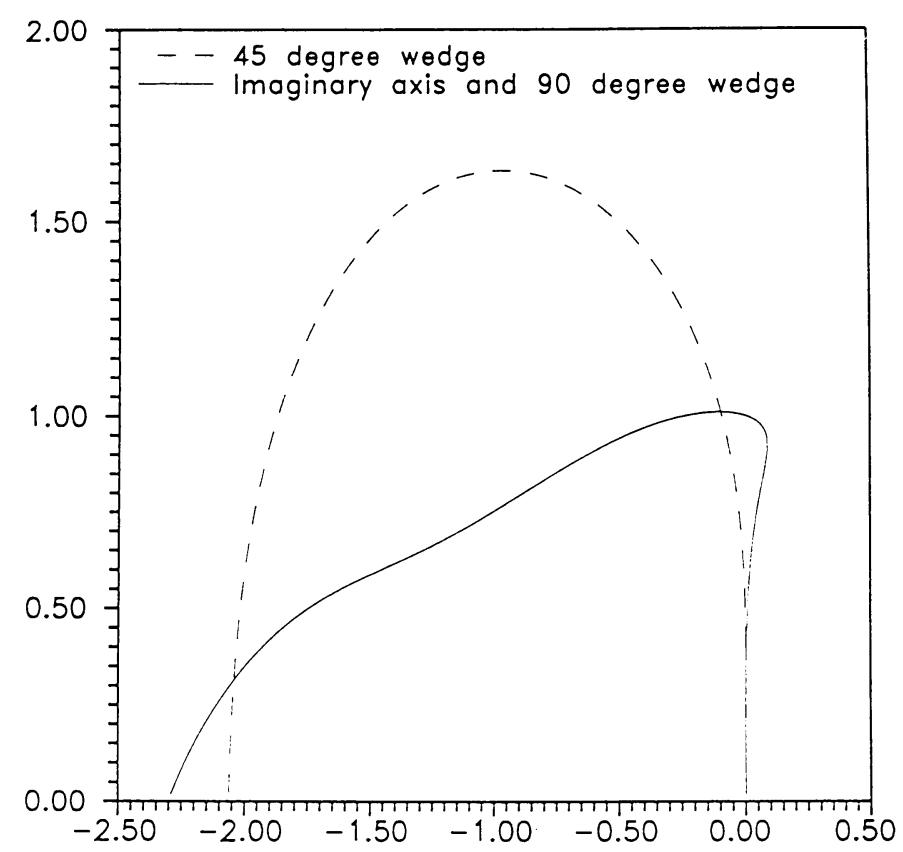

FIGURE 2a

Stability regions for $k=2, m=2, p=3$ schemes

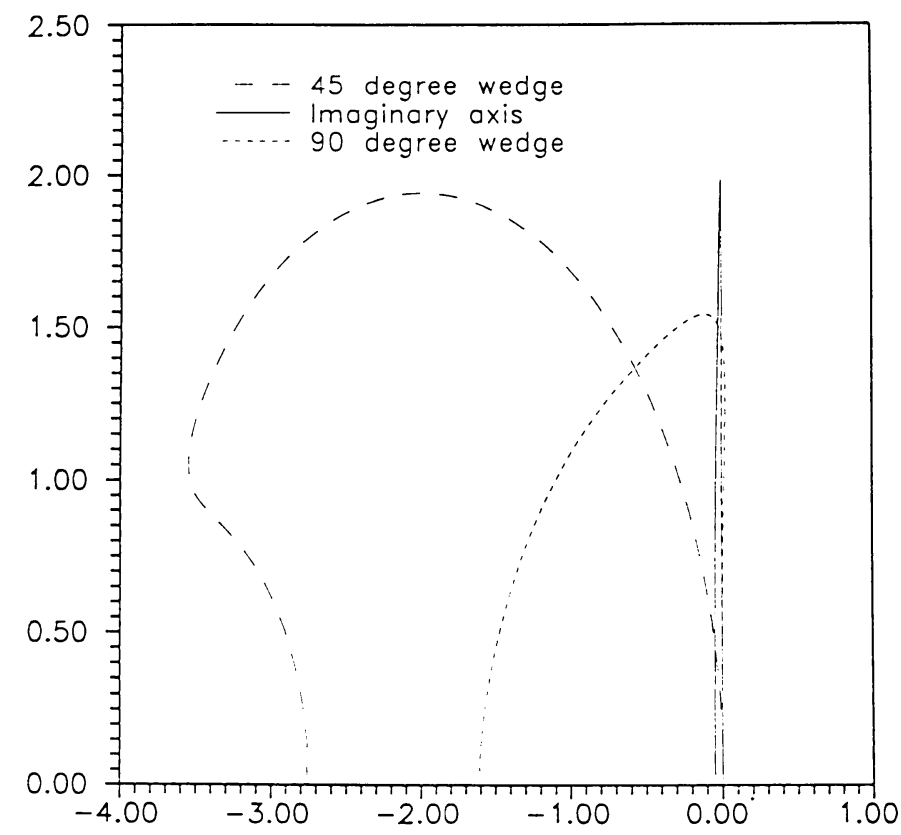

FIGURE $2 \mathrm{~b}$

Stability regions for $k=2, m=2, p=2$ schemes 


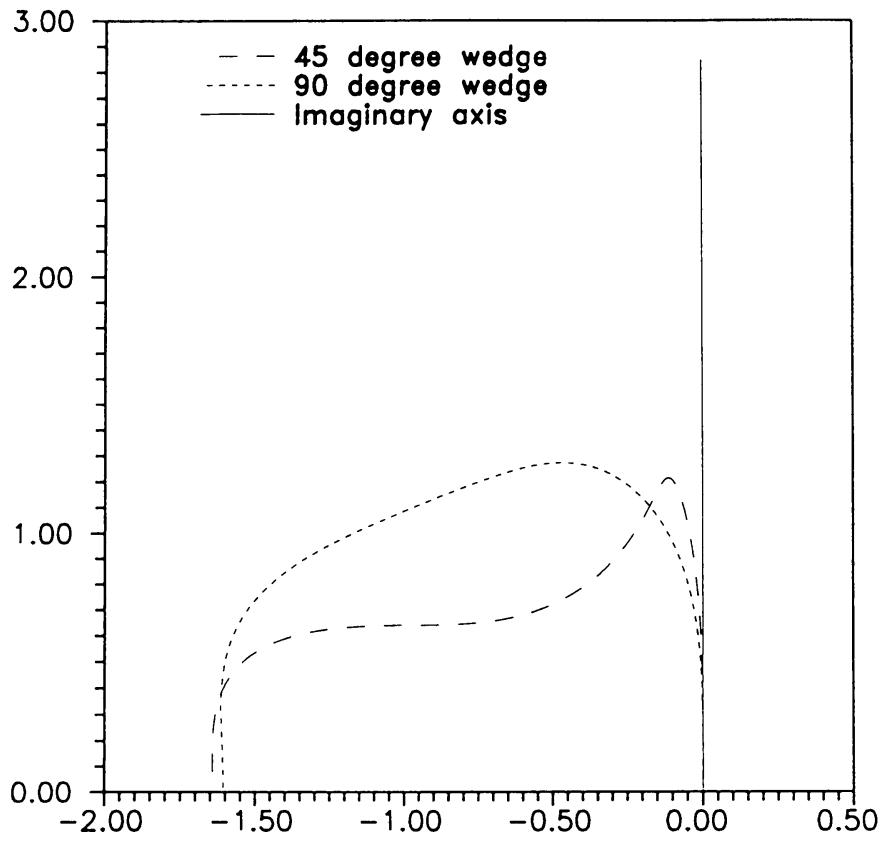

FIGURE 2c

Stability regions for $k=2, m=3, p=3$ schemes

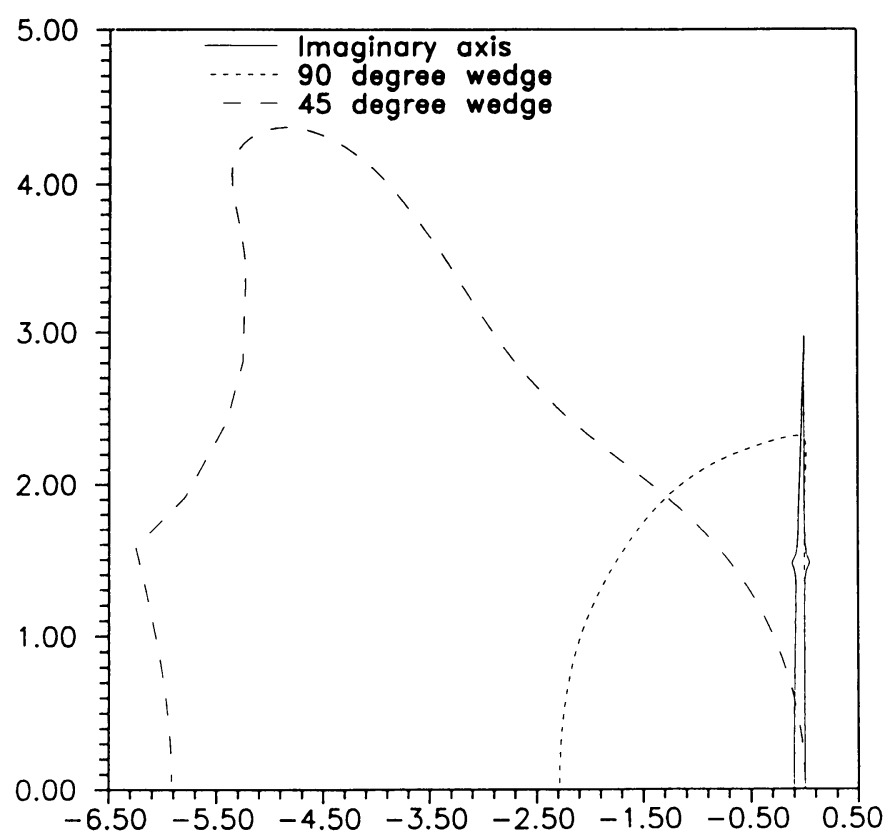

FIGURE 2d

Stability regions for $k=2, m=3, p=2$ schemes 
curves are different in each case, and clearly it is these that have dictated the optimization. Indeed, the other results show that the smaller wedges allow larger maximal radii. We conclude that the optimization procedure is basically successful. Currently, we are communicating with Nick Gould of Harwell about the use of new software, not yet publicly available, which may improve the results and simplify the optimization procedure. Examples of the stability regions are given in Figures $2 a-2 d$. Note that we do not use the same scale on both axes but prefer to compare regions for a chosen method. When both axes have the correct scale, the wedges fit as required.

As already noted in the previous section, there is a two-step, three-stage scheme of order of accuracy $p=3$, that has $\beta_{\text {imag }}=2.84$. This appears, at first glance, to contradict the result of Jeltsch and Nevanlinna [8]. Their scheme is given by $P(z)=1, S(z)=2 z+\frac{8}{27} z^{3}$ and has $\beta_{\text {imag }}=1.5$ because there is a double root at $z=1.5 i$. Perturbing $S(z)$ slightly gives a third-order scheme, with

$$
S(z)=2 z+\frac{1}{3} z^{3}
$$

and $\beta_{\text {imag }}=2.8473$. The root locus of $z^{3}+6 z-6 i \sin t=0$ has a branch point at $z=i \sqrt{2}$ for $t=\cos ^{-1}(1 / 3)$ and therefore the scheme with $S(z)=$ $2 z+z^{3} / 3$ does not satisfy Property $C$ as defined by Jeltsch and Nevanlinna [9]. Their theory, however, requires that Property $C$ is satisfied. Therefore, our result shows that there are schemes which fall outside the theory of Jeltsch and Nevanlinna [9] but still may be useful. For the Jeltsch and Nevanlinna scheme and the scheme which we propose, the stability region is just an interval along the imaginary axis.

In conclusion, observe that the two-step schemes are generally more efficient than the one-step schemes. An increase in order reduces efficiency, as would be expected. In most cases there is still some freedom left over. Renaut [14] used this freedom to design efficient algorithms for error control. Numerical experiments are currently in progress and will be reported later.

\section{BIBLIOGRAPHY}

1. G. D. Byrne and R. J. Lambert, Pseudo-Runge-Kutta methods involving two points, J. Assoc. Comput. Mach. 13 (1966), 114-123.

2. E. Hairer and G. Wanner, On the Butcher group and general multi-value methods, Computing 13 (1974), 1-15.

3. P. Henrici, Discrete variable methods in ordinary differential equations, Wiley, New York, 1962.

4. P. J. van der Houwen, Construction of integration formulae for initial value problems, NorthHolland, Amsterdam, 1977.

5. A. Iserles and R. A. Williamson, Stability and accuracy of semidiscretised finite difference methods, IMA J. Numer. Anal. 4 (1984), 289-307.

6. Z. Jackiewicz, R. Renaut, and A. Feldstein, Two-step Runge-Kutta methods (submitted).

7. A. Jameson, Transonic aerofoil calculations using the Euler equations, Numerical Methods for Aeronautical Fluid Dynamics (P. L. Roe, ed.), Academic Press, 1982. 
8. R. Jeltsch and O. Nevanlinna, Stability of semidiscretizations of hyperbolic problems, SIAM J. Numer. Anal. 20 (1983), 1210-1218.

9. Stability and accuracy of time discretizations for initial value problems, Numer. Math. 40 (1982), 245-296.

10. I. P. E. Kinmark and W. G. Gray, One step integration methods of third-fourth order accuracy with large hyperbolic stability limits, Math. Comput. Simulation 26 (1984), 181188.

11. J. D. Lawson, An order five Runge-Kutta process with extended region of stability, SIAM J. Numer. Anal. 3 (1966), 593-597.

12. J. H. Miller, On the location of zeros of certain classes of polynomials with application to numerical analysis, J. Inst. Math. Appl. 8 (1971), 397-406.

13. J. Pike and P. L. Roe, Accelerated convergence of Jameson's finite volume Euler scheme using van der Houwen integrators, Comput. \& Fluids 13 (1985), 223-236.

14. R. A. Renaut-Williamson, Numerical solution of hyperbolic partial differential equations, Ph.D. thesis, Cambridge University, England, 1985.

Department of Mathematics, Arizona State University, Tempe, Arizona 85287-1804 E-mail address: ATRZR@ASUACAD.BITNET 